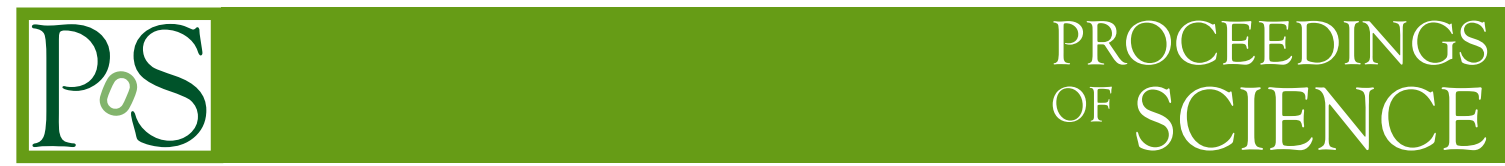

\title{
Review of Flavour Physics
}

\author{
Federico Mescia* \\ Universitat de Barcelona \\ E-mail: federico.mesciaegmail.com
}

I will present a summary of what we learned so far from low-energy flavor observables, concerning on physics beyond the Standard Model (SM). In the past few years there has been a great experimental progress in quark and lepton flavour physics. In the quark sector, the validity of the SM has been strongly reinforced by a series of challenging tests. As I try to show, looking for physics beyond the SM via the Flavour Window is still a powerful tool thanks also to forthcoming results from LHC and future B Factories.

Frontiers of Fundamental Physics 14 - FFP14,

15-18 July 2014

Aix Marseille University (AMU) Saint-Charles Campus, Marseille

\footnotetext{
${ }^{*}$ Speaker.
} 